\title{
Detection of Glasses Sliding State And Automatic Adjustment
}

\author{
Kai Lin, Hao Hu, SiYi Zhang, Pei Zhang, Wei Yang \\ Beijing University of Posts and Telecommunications, Beijing, 100876, China \\ linkai@bupt.edu.cn;huhao@bupt.edu.cn;zhangsiyi@bupt.edu.cn;zp_163workmail@163.com \\ yangwei@bupt.edu.cn
}

Keywords: Glasses structure, Smart glasses, Angle transducer

\begin{abstract}
This paper introduces an improvement of traditional glasses, which is combined with some sensors and motors that can detect the falling state and make auto adjustment. This product focuses more on the user's comfort comparing with other smart glasses. The passage also demonstrates the principle of the product and gives the test results of the product. It can be seen that if this technology is actually used in the industry, it will fully reform the traditional glasses market.
\end{abstract}

\section{Introduction}

As more and more people wear glasses, the problems of traditional glasses become obvious. One of the most important issues is falling of glasses. According to preliminary statistics, people with poor eyesight raise glasses about ten times a day, or even much more than this number, especially for someone who is fond of exercise, wearing glasses without any anti-skid measures, need to constantly raise glasses. Although this is only a relatively simple action, it will greatly affect people's concentration, particularly when both hands are busy, the glasses will bring great inconvenience. The project intends to integrate "automated" equipment into the glasses to detect and automatically adjust the falling state of the glasses in real time. This is an innovation of traditional glasses, which also has strong practicality.

\section{Related Work}

There are few related companies studying the project within the globe. Although there are many companies that research and develop smart glasses-related products, their focus is on the smart glasses, just like putting glasses on an intelligent system to seize the smart glasses market. For example, Google has launched Google glasses, etc. Since smartphones and other smart devices have mature technologies and a good UI experience, there is not much demand for smart glasses, and sales of Google glasses are not optimistic. Google claimed on September 14, 2015 that it will be equipped with intelligent adjustment systems for its smart glasses, but no mature product has been introduced so far.

\section{Model Design}

Overall Design. Our model can split into 2 parts. First is the detection of the falling state, second is the automatic adjustment of the glasses [1]. Figure 1 shows the overall design of our model.

This model can achieve the basic requirements of glasses adjustment, which is realized by JY-61 sensors and micro step motor. Also, its sensitivity and reaction time can change manually. The following parts give the details of the 2 main modules. 


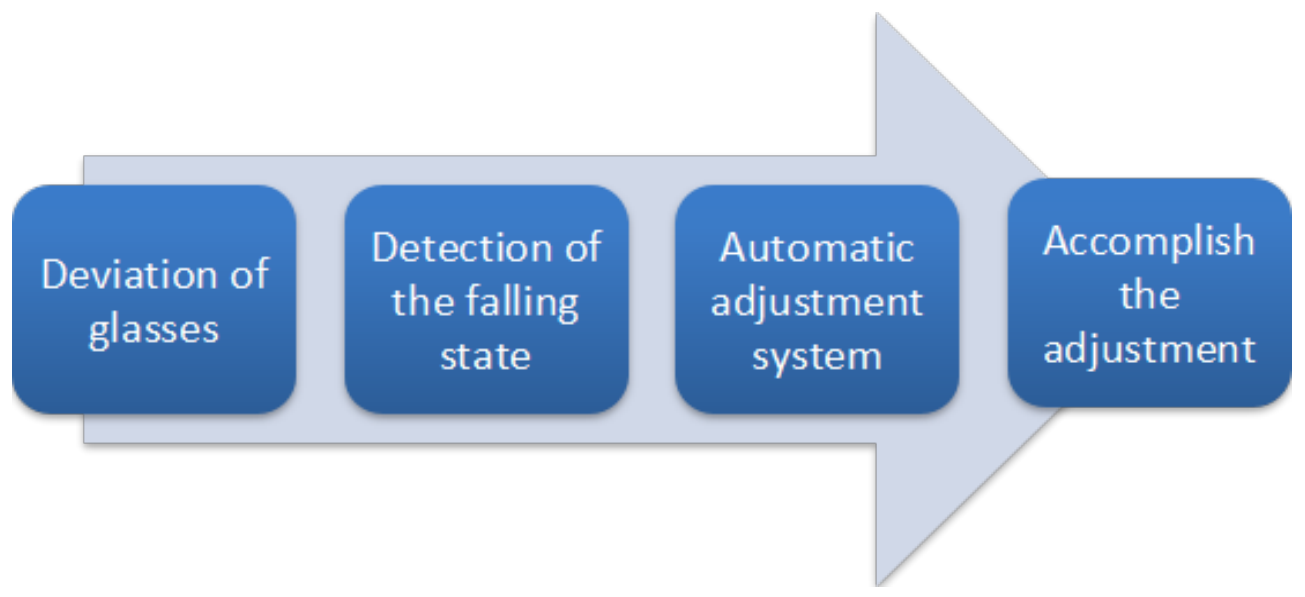

Figure 1: Model design

Detection of the falling state module. This module use two angle transduce to measure the angle of the glasses and the angle of the human face, so that it can calculate the relative difference value of the two angles, to detect whether the glasses have fallen or not. There are 3 directions of angle, $\mathrm{x}, \mathrm{y}$ and $\mathrm{z}$. On our model, we can ignore the $\mathrm{x}$ direction because it will make little influence on the detection of the falling state. So we only take $y$ and $z$ direction to implement our design.

The tolerance of the maximum y and z direction can be set manually. In our model, we took the tolerance of maximum of 4 degrees of $Y$ differences, and 2 degrees of $Z$ differences, which can make the general function used and don't make the system too sensitive.

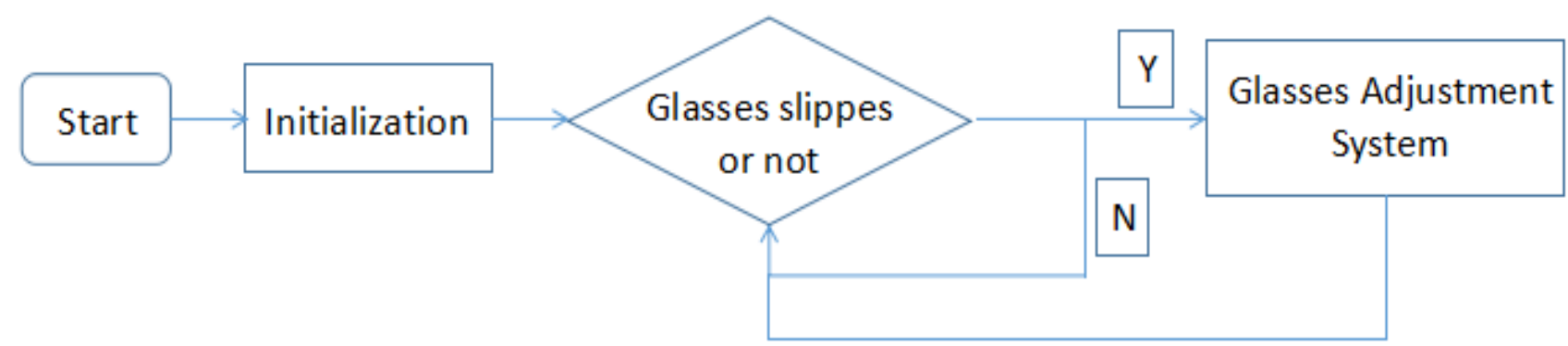

Figure 2: Detection of the falling state module

Auto adjustment system. The mechanism of adjustment is easily comprehensive. Suppose that there is a coordinate system with horizontal axis and vertical axis, which has an origin in conformity with initial place where user wears glasses [2]. In practice, a knob of high friction coefficient and being rotatable and displaceable is placed behind the glasses. When the offset occurs, which indicates that the values in both axes are no longer nil, after being detected by system the knob executes a backward rotation when the specified signal received. With the assist of friction, the glasses will be pulled upwards in the meantime to complete the adjustment process until the values in both axes back to nought (the proximity state of sensor is nil).

The electric motor is used to receive and analyze the output signal from the sensor. After the signal is well analyzed and processed, the Module 1, one of the motor systems, initiate to perform Rough Adjustment, the Module 2 starts to perform Fine Adjustment t afterwards.

While deviation comes up, the Rough Adjustment comes into force to cope with the decrease of huge displacement in $\mathrm{x}$-axis and $\mathrm{y}$-axis [3]. Fine Adjustment can pull the glasses backwards 
minutely while the sensor feels close to human face, which is reached via two Micro Slow Motors in both sides [4].

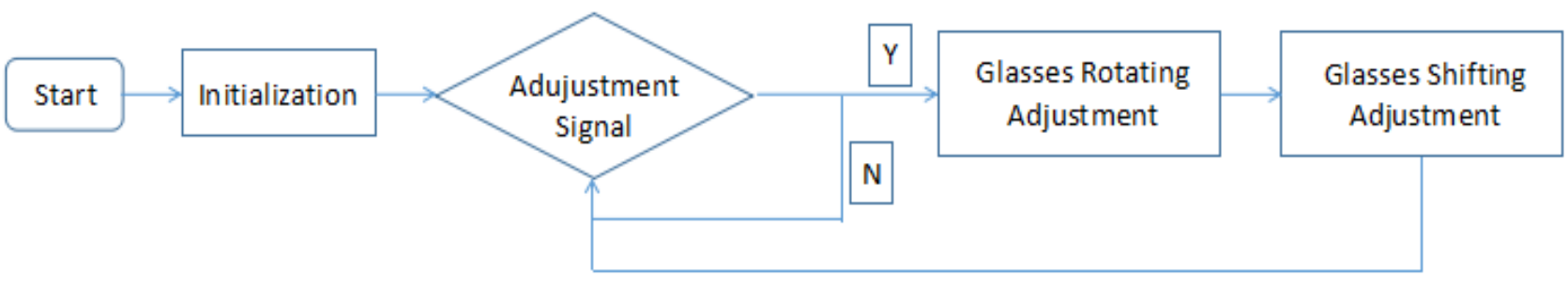

Figure 3: Auto Adjustment System

\section{Result of the Experiment}

We took the tolerance of maximum of 4 degrees of $\mathrm{Y}$ differences, and 2 degrees of $\mathrm{Z}$ differences as parameter, and have many tests on our model [5], more than 90 percent of them fit the imagination; rest of them was caused by some system error, such as the bad contact of wires.

Figure 2 gives some classic results of our tests.

\begin{tabular}{|c|c|c|c|c|c|}
\hline $\begin{array}{c}\text { Initial Y angle } \\
\text { differences }\end{array}$ & $\begin{array}{c}\text { Initial Z angle } \\
\text { differences }\end{array}$ & $\begin{array}{c}\text { Final Y } \\
\text { angle } \\
\text { differences }\end{array}$ & $\begin{array}{c}\text { Final Z angle } \\
\text { differences }\end{array}$ & Adjust state & $\begin{array}{c}\text { Adjust } \\
\text { time }\end{array}$ \\
\hline $9.52^{\circ}$ & $4.15^{\circ}$ & $2.01^{\circ}$ & $1.88^{\circ}$ & Adjust & $9.2 \mathrm{~s}$ \\
\hline $6.27^{\circ}$ & $3.15^{\circ}$ & $1.31^{\circ}$ & $1.28^{\circ}$ & Adjust & $7.0 \mathrm{~s}$ \\
\hline $3.24^{\circ}$ & $-5.15^{\circ}$ & $3.24^{\circ}$ & $-1.21^{\circ}$ & Adjust & $3.1 \mathrm{~s}$ \\
\hline $2.15^{\circ}$ & $1.22^{\circ}$ & $2.15^{\circ}$ & $1.22^{\circ}$ & Not adjust & - \\
\hline
\end{tabular}

Figure 4: Classic tests results

\section{Conclusion}

In summary, our product is different from traditional smart glasses products on the market, it can detect the state of the glasses falling and make correction, almost all people who wear glasses is in need of this product, which can automatically adjust the position, this will redefine the lifestyle of the person wear glasses. Even though there are some drawbacks of our existing products, such as the overall appearance is not small enough, which may be a burden nowadays. However, if this technology can be promoted in the future, it will surely have a broad market.

\section{Acknowledgements}

This work is supported by Research Innovation Fund for College Students of Beijing University of Posts and Telecommunications.

\section{References}

[1] D. R. Uhlmann and N. J. Kreidl, Glass: Science and Technology / Vol.5, Elasticity and Strength in Glasses. New York; London; Academic Press, 1980.

[2] E. Bolthausen and A. Bovier, Spin Glasses. Berlin: Springer, 20071900; 1900.

[3] M. Margolis, Arduino Cookbook. (2nd; 2; ed.) Farnham: O'Reilly, 2011; 2012. 
[4] J. C. Phillips, "Stretched exponential relaxation in molecular and electronic glasses," Reports on Progress in Physics, vol. 59, pp. 1133, 1996.

[5] J. A. Mydosh 1938, Spin Glasses: An Experimental Introduction. London;Washington, DC; Taylor \& Francis, 1993. 